\title{
VALIDATION OF VitBOD, A SIMPLE BURDEN OF DISEASE INSTRUMENT FOR VITILIGO
}

\author{
Sujeeta Silwal ${ }^{1} \mathrm{MS}$, Vaneeta $\mathrm{Sheth}^{2}, \mathrm{MD}$ \\ ${ }^{1}$ Physiologic Division, Brigham and Women's Hospital, Boston, MA \\ ${ }^{2}$ Clinical Research Program, Department of Dermatology, Brigham and Women's \\ Hospital, Boston, MA
}

Corresponding author: Vaneeta M. Sheth, M.D.

Department of Dermatology

Brigham and Women's Hospital

221 Longwood Ave

Boston, MA 02115

Phone 617-732-4918, Fax: 617-582-6060

E-mail: vaneeta.sheth@gmail.com

The authors have no conflicts of interest to declare. This study was approved by the institutional review board

\section{ABSTRACT}

Background: Vitiligo is a chronic, progressive condition of skin depigmentation that has a negative impact on quality of life.

Aim of the study: To validate a novel, low-burden tool to more accurately assess the burden of disease on those affected.

Methods: The VitBOD was designed as a ten question tool using visual analog scales to assess various physical, mental and emotional domains of health specific to vitiligo. 90 subjects with dermatologist-confirmed vitiligo agreed to complete the self-administered VitBOD questionnaire along with the previously validated Short Form-36 (SF-36).

Results: 67 fully completed questionnaires were returned. VitBOD scores were transformed to fit the direction of the SF-36 for statistical analysis. Median overall scores were significantly lower for VitBOD as shown by the paired t-test (75.3 vs 85, $\mathrm{p}=0.015)$. VitBOD showed high internal consistency (Cronbach's alpha $=0.9)$. Vitality, social, and mental health domains correlated positively with SF-36.

Conclusion: VitBOD can reliably measure burden of disease in vitiligo as well as the SF-36 with distinct advantages being ease and speed of use along with disease-specificity. Testing in broader patient populations would be useful for further validation.

Keywords: vitiligo; quality of life; burden of disease; mental health 


\section{INTRODUCTION}

Skin color, the most visible feature of one's body, has historically had a significant influence on an individual's social position defined by wealth, health, worth and attractiveness (1). Vitiligo, a skin disorder of progressive depigmentation caused by the loss of melanocytes in the affected areas, is one of the most common diseases to affect skin color. Although the statistics on vitiligo vary, it is generally considered to affect at least $1-2 \%$ of the world's population (2). While it is not considered a life threatening condition and does not cause physical impairment, vitiligo can have a substantial negative impact on patients' social and psychological wellbeing, especially in darker - skinned individuals (3-5).

In dermatology, the three most widely used tools to measure quality of life (QOL) are Skindex-16 (6), the Dermatology Life Quality Index (DLQI) (7), and the Dermatology-Specific Quality of Life Questionnaire (DSQLQ) (8). In recent years, two additional vitiligospecific QOL instruments, VitiQOL (9) and vitiligo impact scale-22 (VIS-22) $(10,11)$ have been developed and validated using DLQI and Skindex-16. These tools have shown that vitiligo significantly affects QOL. General health measures such as short form-36 (SF-36) (12) serve as a gold standard to assess several domains, including symptoms, disability, emotion and function, and many studies have reported significant effects of disease on QOL using SF-36 $(13,14)$. However, while a generic instrument such as SF-36 is useful in measuring the overall impact of disease, it cannot elucidate diseasespecific concerns, especially as relates to skin disease (15). It is also time consuming to administer. Vitiligo-specific instruments are necessary to understand the unmet needs of this patient population because vitiligo is an asymptomatic disease with significant psychosocial impairment. While the DLQI, VitiQOL and VIS-22 have given us an understanding of the extent to which QOL is affected in vitiligo patients, they can also be more challenging to use in routine care. Furthermore, they rely on scales with lower discriminatory ability due to the limited number of answer choices.

To address the need for a simpler tool that could reliably measure vitiligospecific burden of disease, we designed the Vitiligo Burden of Disease (VitBOD) questionnaire based on the visual analog scale. The aim was to focus on recognizing the emotional and psychological burden of disease with less emphasis on the impact of physical symptoms. We then validated VitBOD by comparing with responses to the SF-36.

\section{MATERIALS AND METHODS}

VitBOD was designed as a selfadministered tool based on the eight domains of health as outlined in the ShortFrom 36: physical functioning, rolephysical, bodily pain, general health, vitality, social functioning, role emotional and mental health (12). Disease-specific questions were created through an $\mathrm{e}$ Delphi process using patient interviews and discussions with clinicians specializing in vitiligo. A visual analog scale was used where subjects were asked to mark their response to each item by placing an " $\mathrm{X}$ " on a $10 \mathrm{~cm}$ linear scale where 0 is represented by 'Never' and 10 by 'Always' (Figure 1). A score of 0 represents no burden of disease and a score of 100 represents very high burden of disease. For comparison, subjects were also given the SF-36 to complete. The 
scores for the SF-36 were summed and transferred on to a scale of 0 (worst health) to 100 (best health) using the SF-Health
Scoring Software. For statistical analysis, the inverse of VitBOD scores was used to match the direction of the SF-36.

Figure 1. VITILIGO BURDEN OF DISEASE (VITBOD) QUESTIONNAIRE:

Please check ALL that applies:

\begin{tabular}{|l|l|l|l|}
\hline Hospital: & & Gender: & Male / Female \\
\hline Marital Status: & $\begin{array}{l}\text { Married, Single, } \\
\text { Divorced, Widowed }\end{array}$ & Race: & $\begin{array}{l}\text { Caucasian, Black, Asian, Latino / } \\
\text { Hispanic, Native American, Other }\end{array}$ \\
\hline
\end{tabular}

\section{Quality of Life:}

Please answer the questions below by indicating how much your vitiligo skin condition has bothered you. Please mark your response on the black lines with an $\mathbf{X}$

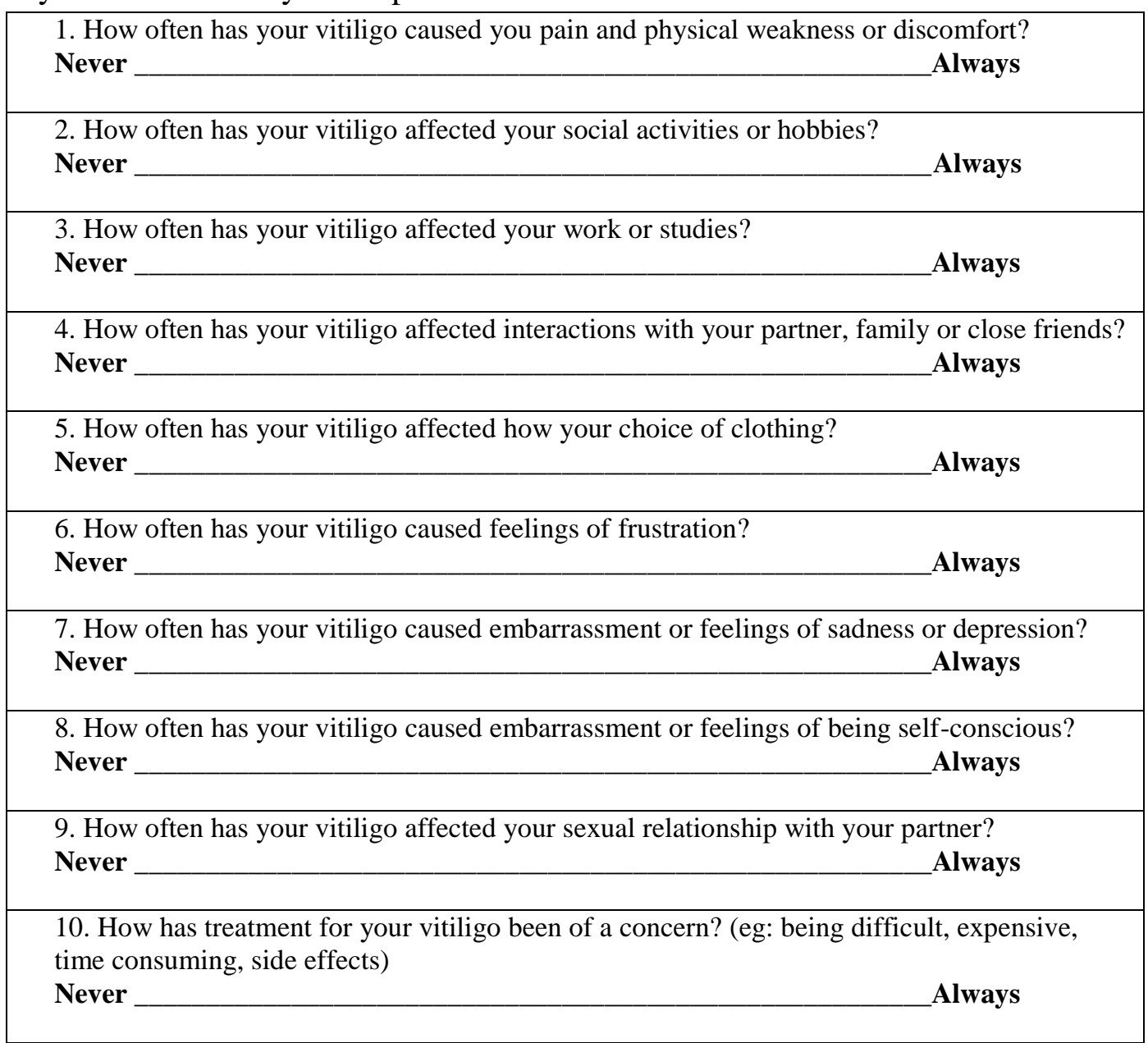

What bothers you the most about your vitiligo skin condition?

Study participants:

Subjects with vitiligo were identified initially using the diseasespecific ICD-9 diagnosis code 709.01. Patient medical records were also reviewed to confirm a diagnosis of vitiligo for dermatology outpatients who chose to participate in the study. The VitBOD questionnaire was validated in English; however, Spanish-speaking patients could participate if an interpreter was present. Inclusion criteria consisted of subjects being 18 years of age or older and having a dermatologist-confirmed diagnosis of vitiligo. Exclusion criteria included 
subjects less than 18 years of age and subjects whose diagnosis of vitiligo could not be confirmed by chart review or conferring with the treating dermatologist. Ninety patients agreed to participate in this study. Of these, 67 participants fully completed both questionnaires (completion rate $=74.4 \%)$.

\section{Statistical analysis:}

Analysis was performed on the 67 fully completed questionnaires using SPSS software (Statistical Package for the Social Sciences, version 11.0, SPSS, Inc., Chicago, IL, USA). Descriptive statistics were used to summarize the data. We used paired t-tests for differences between the overall median scores on the two questionnaires. Spearman's correlation coefficients were used to study the correlation between SF-36 and VitBOD questions. VitBOD was also evaluated for internal consistency and reliability using Cronbach's alpha and comparing with scores from the SF-36 survey.

\section{RESULTS}

Demographics:

We analyzed data from the 67 subjects who fully completed both questionnaires. The mean age of participants was 45. Respondents were $61 \%$ female and $39 \%$ male. White/Caucasian was the highest represented race for both genders $(55.6 \%)$ followed by Black (12.2\%). Asian and Other races respectively were $11.1 \%$ and $8.9 \%$ with the least number seen for Latino/Hispanic at $6.7 \%$. $55.6 \%$ of respondents were married, $34.4 \%$ single, $5.6 \%$ divorced and $2.2 \%$ widowed.

\section{Validation:}

In comparing VitBOD to the SF36, the overall median transformed VitBOD score was 75.3 compared to an overall median score of 85 on the SF-36. Paired t-test was used to evaluate the differences between median VitBOD scores and median SF-36 scores, revealing a significant difference of $-9.8(\mathrm{p}=0.015)$. VitBOD scores being lower than SF-36 scores suggests better ability to measure disease-specific impact on participants. Overall VitBOD scores were not normally distributed but rather were left-skewed, suggesting that scores tended to be weighted towards the higher end of the scale.

Figure 2: Scatterplot of median VITBOD scores and median SF-36 scores

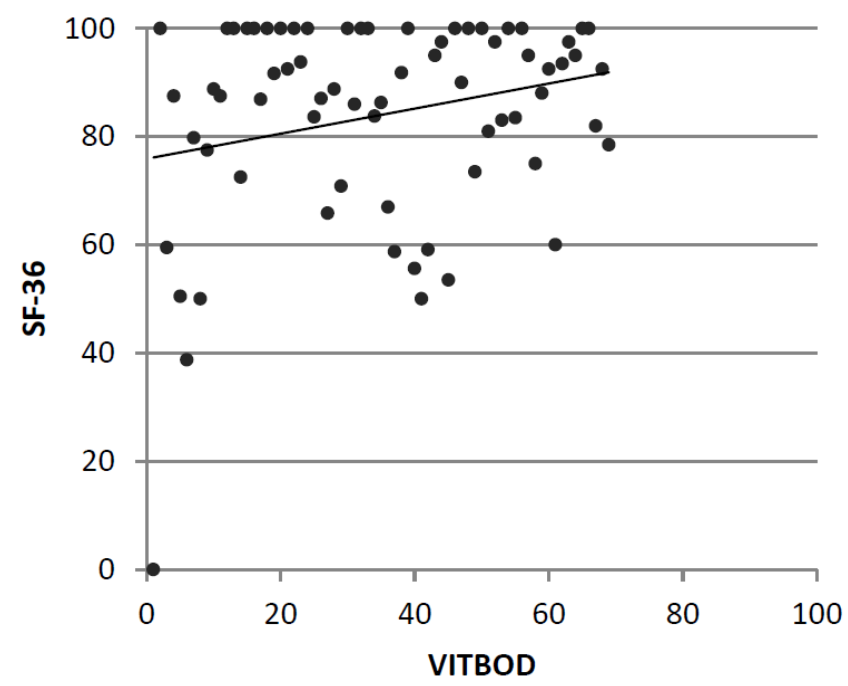


Individual VitBOD questions were then analyzed for correlation with the eight domains of health as measured by the SF-36: physical functioning, role physical, bodily pain, general health, vitality, social functioning, role emotional and mental health. We found that although
VITBOD scores did not correlate with all eight domains of SF-36, bodily pain correlated positively with question 1 , and the domains of vitality, social functioning and mental health correlated positively with questions 6 through 8 (Table 1).

Table 1: Spearman's Correlation Coefficients for VITBOD items as compared to SF-36 domains

\begin{tabular}{l|r|r|r|r|r|r|r|r}
\hline & \multicolumn{7}{|c}{ SF-36 Domain } \\
\hline $\begin{array}{l}\text { VITBOD } \\
\text { Question }\end{array}$ & $\begin{array}{r}\text { Physical } \\
\text { Functioning }\end{array}$ & $\begin{array}{r}\text { Role } \\
\text { Physical }\end{array}$ & $\begin{array}{r}\text { Body } \\
\text { Pain }\end{array}$ & $\begin{array}{r}\text { General } \\
\text { Health }\end{array}$ & Vitality & $\begin{array}{r}\text { Social } \\
\text { Functioning }\end{array}$ & $\begin{array}{r}\text { Role } \\
\text { Emotional }\end{array}$ & $\begin{array}{r}\text { Mental } \\
\text { Health }\end{array}$ \\
\hline 1 & 0.03 & 0.12 & $\mathbf{0 . 2 6}$ & 0.10 & 0.08 & 0.19 & 0.03 & 0.05 \\
\hline 2 & -0.14 & -0.10 & -0.06 & 0.11 & 0.23 & 0.12 & -0.01 & 0.20 \\
\hline 3 & $-0.30^{*}$ & -0.04 & -0.03 & -0.02 & 0.09 & 0.14 & 0.02 & 0.01 \\
\hline 5 & $-0.35^{*}$ & -0.14 & $-0.25^{*}$ & 0.05 & 0.19 & 0.13 & -0.02 & 0.12 \\
\hline 6 & -0.16 & -0.01 & -0.05 & -0.01 & 0.14 & 0.13 & -0.03 & 0.11 \\
\hline 8 & $-0.28^{*}$ & 0.04 & -0.04 & 0.07 & $\mathbf{0 . 3 7}$ & $\mathbf{0 . 3 0}$ & 0.21 & $\mathbf{0 . 3 8}$ \\
\hline 9 & -0.23 & 0.04 & -0.08 & 0.08 & $\mathbf{0 . 3 1}$ & $\mathbf{0 . 3 1}$ & 0.17 & $\mathbf{0 . 3 9 *}$ \\
\hline 10 & -0.21 & 0.03 & -0.07 & 0.04 & $\mathbf{0 . 4 0}$ & $\mathbf{0 . 3 6}$ & 0.14 & $\mathbf{0 . 4 0} *$ \\
\hline & -0.16 & -0.04 & -0.07 & 0.05 & 0.22 & 0.20 & 0.15 & 0.23 \\
\hline
\end{tabular}

*p-value $<0.05$

Table 1. VITBOD scores positively correlated with SF-36 domains of body pain, vitality, social functioning, role emotional and mental health.

The Cronbach's alpha reliability test revealed strong internal consistency and reliability between VitBOD questions (overall Cronbach's alpha $=0.9$ ). The lowest item scale correlation was seen in question 1 regarding physical weakness which is consistent with the concept that vitiligo does not cause significant physical impairment (Table 2).

Table 2: Item scale correlations and Cronbach's alpha for VITBOD

\begin{tabular}{l|r|r}
\hline VITBOD question & Item-scale correlation & Alpha \\
\hline 1 & 0.337 & 0.907 \\
\hline 2 & 0.770 & 0.882 \\
\hline 3 & 0.430 & 0.902 \\
\hline 4 & 0.651 & 0.891 \\
\hline 5 & 0.692 & 0.888 \\
\hline
\end{tabular}




\begin{tabular}{l|l|l}
\hline 7 & 0.869 & 0.874 \\
\hline 8 & 0.848 & 0.875 \\
\hline 9 & 0.495 & 0.899 \\
\hline 10 & 0.553 & 0.897 \\
\hline
\end{tabular}

Overall Cronbach's alpha $=0.90$

Table 2. VITBOD showed excellent internal consistency with an overall Cronbach's alpha of 0.9 , with highest item-scale correlation seen for questions 6,7 , and 8 assessing disease impact on emotional and mental health factors.

Examining VitBOD scores alone, we did not find any significant difference in overall scores based on gender, race/ethnicity, or marital status (Table 3). The same results were seen when looking at just SF-36 scores. We then subdivided respondents into those who had vitiligo on just exposed skin (defined as face, neck, hands, and/or feet), just unexposed skin (other remaining body sites), or those who had both areas affected. Analysis of responses based on location of lesions also did not reveal any significant difference on VitBOD scores or SF-36 scores between the three categories.

Table 3: Median VITBOD and SF-36 scores by Demographics

\begin{tabular}{|c|c|c|}
\hline & Mean (Std Deviation) & p-value \\
\hline \multicolumn{3}{|l|}{ Median VITBOD } \\
\hline \multicolumn{3}{|l|}{ Gender } \\
\hline Male & $79.2(24.7)$ & 0.368 \\
\hline Female & $72.7(31.1)$ & \\
\hline \multicolumn{3}{|l|}{ Race/Ethnicity } \\
\hline White & $77.0(27.3)$ & 0.554 \\
\hline Non-white & $72.7(31.1)$ & \\
\hline \multicolumn{3}{|l|}{ Marital Status } \\
\hline Married & $76.2(29.4)$ & 0.675 \\
\hline Not married & $73.1(28.3)$ & \\
\hline \multicolumn{3}{|l|}{ Location } \\
\hline Both & $71.3(29.1)$ & 0.107 \\
\hline Just Exposed & $89.3(18.5)$ & \\
\hline Just Unexposed & $70.5(39.2)$ & \\
\hline \multicolumn{3}{|l|}{ Median SF-36 } \\
\hline \multicolumn{3}{|l|}{ Gender } \\
\hline Male & $85.9(19.5)$ & 0.771 \\
\hline Female & $84.6(13.8)$ & \\
\hline \multicolumn{3}{|l|}{ Race/Ethnicity } \\
\hline White & $87.8(13.3)$ & 0.092 \\
\hline Non-white & $80.9(18.5)$ & \\
\hline \multicolumn{3}{|l|}{ Marital Status } \\
\hline Married & $85.9(15.8)$ & 0.517 \\
\hline
\end{tabular}




\begin{tabular}{l|r|r}
\hline Not married & $83.1(16.2)$ & \\
\hline Location & & \\
\hline Both & $84.6(16.6)$ & 0.118 \\
\hline Just Exposed & $92.4(9.2)$ & \\
\hline Just Unexposed & $78.6(78.6)$ & \\
\hline
\end{tabular}

\section{DISCUSSION}

Findings in our study suggest that there is a good correlation between VITBOD and SF-36 in terms of overall scores. Most patients, regardless of gender, race/ethnicity and other factors had similar concerns about vitiligo. VitBOD and SF-36 showed a modest level of agreement across four domains of health between the two questionnaires. Furthermore, the internal consistency of VitBOD was very high. The distinct advantage of VitBOD is the ease and speed of administration as compared to currently existing instruments.

However, our study does have several important limitations. Our sample was based on recruitment done at a tertiary care outpatient academic dermatology clinic, which does lead to selection bias and does not mirror the general population of vitiligo patients. This could be overcome in the future by sampling other geographic sites or online distribution of the questionnaire to reach a broader population. Also, one major limitation of our analysis was that the tool was only compared to the SF-36. Given the different nature of questioning in the tools, it was more challenging to correlate the two scores. The validation work would have been stronger had we also tested VitBOD alongside the DLQI and VitiQOL which are more similar in questionnaire design. However, we did find that overall scores had a similar trend and that, when subanalysis for different clinical features of vitiligo were evaluated, there was no difference between trends in SF-36 and VitBOD scores. This suggests a good overall similarity in ability to measure impact of disease. Another limitation in our study was that reproducibility and responsiveness were unable to be evaluated in the short time span of this pilot study but would be important next steps for investigation.

It was interesting to find that in our sample population, there was no difference in overall scores based on location of skin lesions. This is in contradiction to the findings of the VitiQOL questionnaire where having lesions on exposed locations was shown to have greater impact on QOL. We hypothesize that this may be due to validation on a small sample size that is not representative of vitiligo patients as a whole. Another unexpected finding was the left-skewed distribution which suggested higher than expected VitBOD scores. We believe this may in part be explained by the fact that the majority of our patients tended to be highly educated and most were in stable relationships which may have provided a greater level of comfort with their disease regardless of clinical features.

Finally, our instrument does not take into account any financial impact of factors such as missed work days due to disease or treatments such as phototherapy or the cost of therapies, which would be another important aspect of disease burden to consider in future studies.

We believe that an instrument such 
as VitBOD has several practical applications. Firstly, it can serve as an aid to clinicians in identifying patients who may be at higher risk of psychological or emotional difficulty in coping with their diagnosis. By offering these patients counseling, information on support networks and connecting them to other resources, it will allow better overall care of patients and possibly enhance compliance with treatments. Secondly, it can be useful as an outcome measure in clinical trials. By demonstrating that a therapy not only has an effect on repigmentation but also improves the overall burden of disease, it provides a stronger argument for why therapies should be instituted as well as covered by insurers. Finally, tools such as VitBOD, when used in larger populations, can provide clinicians and patients with a wider sense of how dermatologic diseases such as vitiligo (which are readily visible on the skin but generally asymptomatic) truly impact those affected in ways that may not be easily apparent.

\section{CONCLUSION}

Further work to validate the VitBOD in a larger group of patients in comparison to other previously validated tools would be a critical next step to validate VitBOD as an outcome measure. There is a definite need to develop a low burden instrument that can accurately and reliably measure the impact of vitiligo on those affected. In this era of changing health care dynamics, it is crucial that we are able to justify the need to treat these patients as well as show that treatments affect outcomes including quality of life in a positive manner. Doing so will help improve coverage for therapies and quality of care that these patients need.

\section{ACKNOWLEDGEMENTS}

We would like to thank Cara Joyce of Tulane University for her help with statistical analysis. 


\section{REFERENCES}

1. Bhandarkar, SS, Kundu, RV. Quality-of-life issues in vitiligo. Dermatol Clin, 2012; 2: 255-68.

2. Ezzedine K, Eleftheriadou V, Whitton M, van Geel N. Vitiligo. Lancet. 2015; Jan 14.

3. Shankar, DS, Shashikala, K, Madala, R. Clinical patterns of vitiligo and its associated co morbidities: A prospective controlled cross-sectional study in South India. Indian Dermatol Online J 2012; 3(2): 114-8.

4. Wang, KY, Wang, KH, Zhang, ZP. Health- related quality of life and marital quality of vitiligo patients in China. J Eur Acad Dermatol Venereol 2011; 25(4): 429-35.

5. Borimnejad L, Parsa Yekta Z, Nikbakht-Nasrabadi A, Firooz A. Quality of life with vitiligo: comparison of male and female muslim patients in Iran. Gend Med 2006; 3(2): 124-30.

6. Chren MM, Lasek RJ, Sahay AP, Sands LP. Measurement properties of Skindex-16: a brief qualityof-life measure for patients with skin diseases. J Cutan Med Surg 2001; 5(2):105-10.

7. Finlay AY, Khan GK. Dermatology Life Quality Index (DLQI)--a simple practical measure for routine clinical use. Clin Exp Dermatol 1994; 3:210-6.

8. Anderson RT, Rajagopalan R. Development and validation of a quality of life instrument for cutaneous diseases. $J$ Am Acad Dermatol 1997; 37(1):41-50.

9. Lilly, E, Lu, PD, Borovicka, $\mathrm{JH}$, et al. Development and validation of a vitiligo-specific quality-of-life instrument (VitiQoL). J Am Acad Dermatol 2013; 69(1):e11-8.
10. Krishna GS, Ramam M, Mehta M, et al. Vitiligo impact scale: an instrument to assess the psychosocial burden of vitiligo. Indian $J$ Dermatol Venereol Leprol 2013; 79(2):205-10.

11. Gupta V, Sreenivas V, Mehta M, et al. Measurement properties of the Vitiligo Impact Scale-22 (VIS-22), a vitiligo-specific quality-of-life instrument. Br J Dermatol 2014; 171(5):1084-90.

12. Brazier JE, Harper R, Jones $\mathrm{NM}$, et al. Validating the SF-36 health survey questionnaire: new outcome measure for primary care. BMJ 1992; 305(6846):160-4.

13. Kiebert G, Sorensen SV, Revicki D, et al. Atopic dermatitis is associated with a decrement in healthrelated quality of life. Int J Dermatol 2002; 41(3):151-8.

14. Tabolli S1, Mozzetta A, Antinone $\mathrm{V}$, et al. The health impact of pemphigus vulgaris and pemphigus foliaceus assessed using the Medical Outcomes Study 36-item short form health survey questionnaire. Br J Dermatol 2008; 158(5):1029-34.

15. Ahmed A, Leon A, Butler DC, Reichenberg J. Quality of side effects of common dermatological diseases. Semin Cutan Med Surg. 2013; 32(2):101-9. 